\title{
Chemical enrichment from Wolf-Rayet stellar winds
}

\author{
Georges Meynet \\ Geneva Observatory, CH-1290 Sauverny, Switzerland
}

\begin{abstract}
Stellar winds contribute together with supernovae explosions to the chemical enrichment of the interstellar medium. We recall how the metallicity dependence of the stellar winds implies a metallicity dependence of the stellar yields. We show that an increase of the initial angular velocity has different effects than an increase of the mass loss rates. Wolf-Rayet stars appear as important sources of ${ }^{19} \mathrm{~F}$ and ${ }^{26} \mathrm{Al}$. They are the favoured candidates for the ${ }^{22} \mathrm{Ne}$ anomaly observed in the Galactic cosmic ray sources. They may also have injected into the proto-solar nebula short-lived radionuclides as ${ }^{26} \mathrm{Al},{ }^{36} \mathrm{Cl},{ }^{41} \mathrm{Ca}$, ${ }^{107} \mathrm{Pd}$ and ${ }^{205} \mathrm{~Pb}$.
\end{abstract}

\section{Introduction}

Wolf-Rayet stars inject into the interstellar medium great quantities of mass, electromagnetic radiation, energy and momentum. In particular, a part of the mass they eject trough their strong stellar winds is under the form of new chemical species. Thus WR stars contribute to the chemical evolution of the ISM. Now since massive stars contribute also to this enrichment at the end of their nuclear lifetime when they explode in a supernova event, one can wonder wether it does matter if part of these new synthesized species are ejected by the stellar winds prior the supernova explosion. This is the point we investigate in the first section below.

\section{Effects of metallicity on the stellar yields}

The question above may be reformulated in the following way: 'Is there any difference between the stellar yields of massive stars at different metallicities?' Indeed one expects that the higher the initial metallicity, the more intense are the stellar winds (see e.g., Lamers \& Cassinelli 1996).

In Fig. 1, the evolution of the abundances at the surface of $60 \mathrm{M}_{\odot}$ stellar models for different initial metallicities are presented. The models are taken from Charbonnel et al. (1993, for $Z=0.004)$, Schaerer et al. $(1993$, for $Z=0.008)$ and Schaller et al. $(1992$, for $Z=0.020)$. The effects of an increase of the initial metallicity ${ }^{1}$ on the chemical composition of the WR stellar winds are the following ones:

(i) For the WN phase, whatever the initial metallicity one always obtains $\mathrm{C} / \mathrm{N}$

\footnotetext{
${ }^{1}$ equivalent to an increase of the mass loss rates
} 


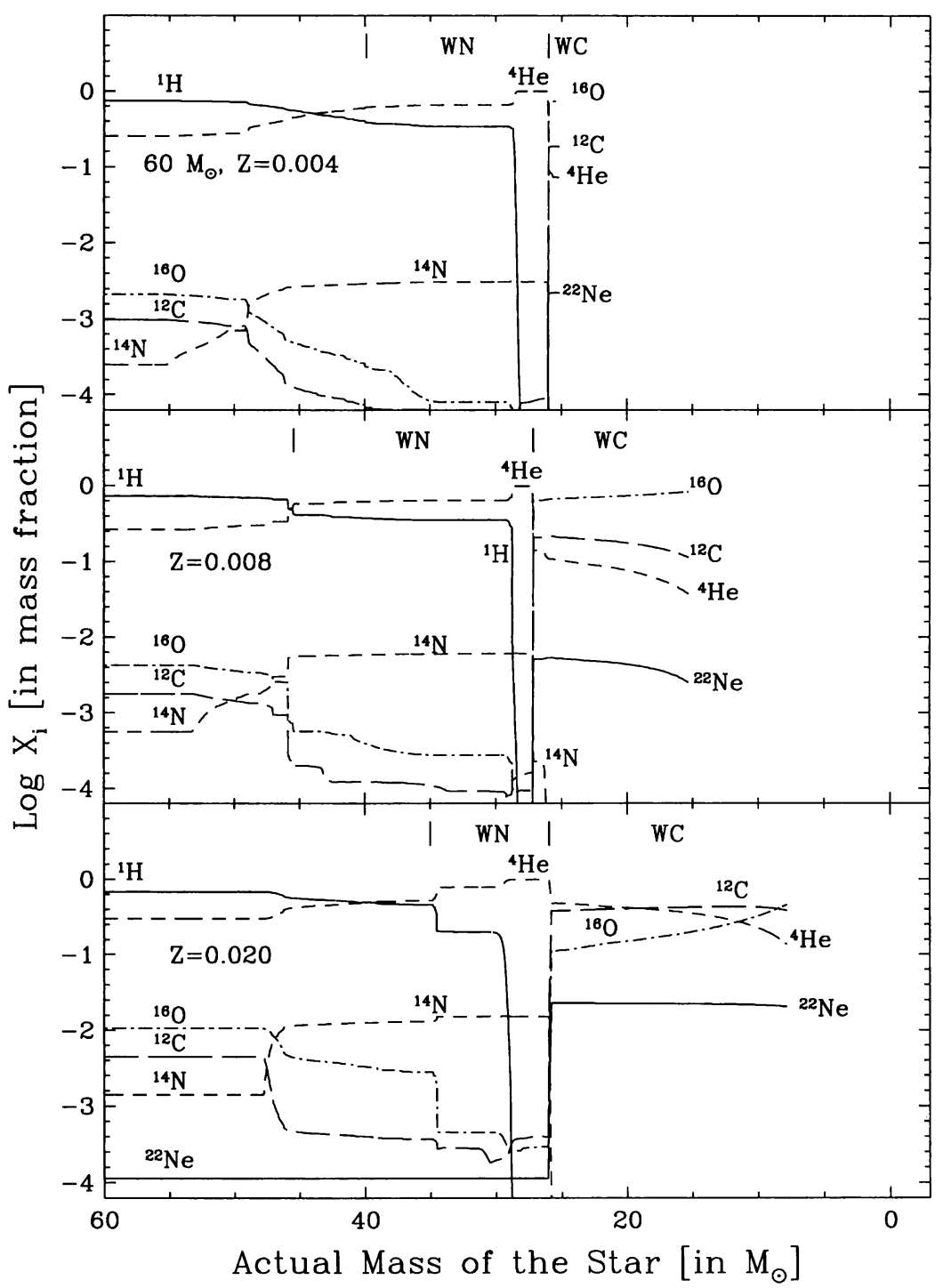

Figure 1. Evolution as a function of the remaining stellar mass of the abundances at the surface of non-rotating $60 \mathrm{M}_{\odot}$ stellar models at different metallicities ( $Z$ is the mass fraction of heavy elements, the solar metallicity corresponds to $Z \simeq 0.020$, see text for more details on the models). The time evolves from left to right (the mass decreases as a result of the stellar winds). The parts of the evolution during which the star may be considered as a WN and a WC star are indicated.

and $\mathrm{O} / \mathrm{N}$ ratios corresponding to nuclear equilibrium values of the $\mathrm{CNO}$ cycle. A change of mass loss has an impact on the quantity of matter ejected during this phase but not on the chemical composition of the matter ejected; and 
(ii) For the WC phase the situation is quite different. The higher the metallicity, the greater the quantities of helium and carbon which are ejected. As explained by Maeder (1991), when the mass loss rate increases, the He-burning core is uncovered at an early stage of its evolution. Therefore the winds remove layers which are still rich in helium and carbon.

This last point has many interesting consequences both for what concerns the stellar yields and the variations of the WC populations with the metallicity.

The effects on the stellar yields have been discussed by Maeder $(1992,1993)$. At low metallicity the new synthesized elements from massive stars are ejected mainly by the supernova explosion and the main product is Oxygen. At high metallicity, stellar winds become the main ejection mechanism for stars with masses above $\sim 30 \mathrm{M}_{\odot}$. These winds are heavily loaded in new helium and carbon. The metallicity dependence of the stellar yields (implied by the metallicity dependence of the mass loss rates) has interesting consequences on our understanding of the origin of some elements in our Galaxy. As an example, according to Prantzos et al. (1994) massive stars could well be the dominant contributors to the carbon enrichement at high metallicity. Let us recall here that when no metallicity dependant yields are used, this carbon enrichment is attributed to low and intermediate mass stars.

Point (ii) above is also the reason why late WC stars are observed only in metal rich regions, while only early WC stars are observed in metal poor zones (Smith \& Maeder 1991).

\section{Effects of rotation}

Fig. 2 presents the evolution of the abundances at the surface of $60 \mathrm{M}_{\odot}$ stellar models for different rotational velocities at solar metallicity (Meynet \& Maeder in preparation). Let us emphasize here that all these models were computed with the Ledoux criterion for convection and with semiconvective mixing (this is the reason why the non-rotating model presented in Fig. 2 is not strictly equivalent to the $Z=0.020$ model shown in Fig. 1, in this last case the Schwarzschild criterion with overshooting was used). The mass loss rates are as in Schaller et al. (1992) multiplied by a factor accounting for the effects of rotation (Friend \& Abbott 1986). Other physical ingredients accounting for the effects of rotation are discussed in André Maeder's talk (these Proceedings).

Are indicated on Fig. 2, the various evolutionary stages through which the star evolves. The criterion to decide when a star enters into the WR phase is the following one : the surface hydrogen abundance must be inferior to 0.4 (in mass fraction) and the effective temperature must be higher than $10000 \mathrm{~K}$. The entrance into the transition phase WN/WC occurs when the mass fraction of carbon becomes higher than one tenth the mass fraction of nitrogen, it ends when the mass fraction of nitrogen becomes inferior than one tenth the mass fraction of carbon.

Adopting these simple citeria, one obtains that rotation implies a mechanism of WR star formation which is different from the non-rotating case. Indeed surface abundances characteristic of the WN stars appear in the rotating model, not as a result of the mass loss which uncovers core layers, but as a result of diffusive mixing in the radiative zones (see also Fliegner \& Langer 1994). The same 


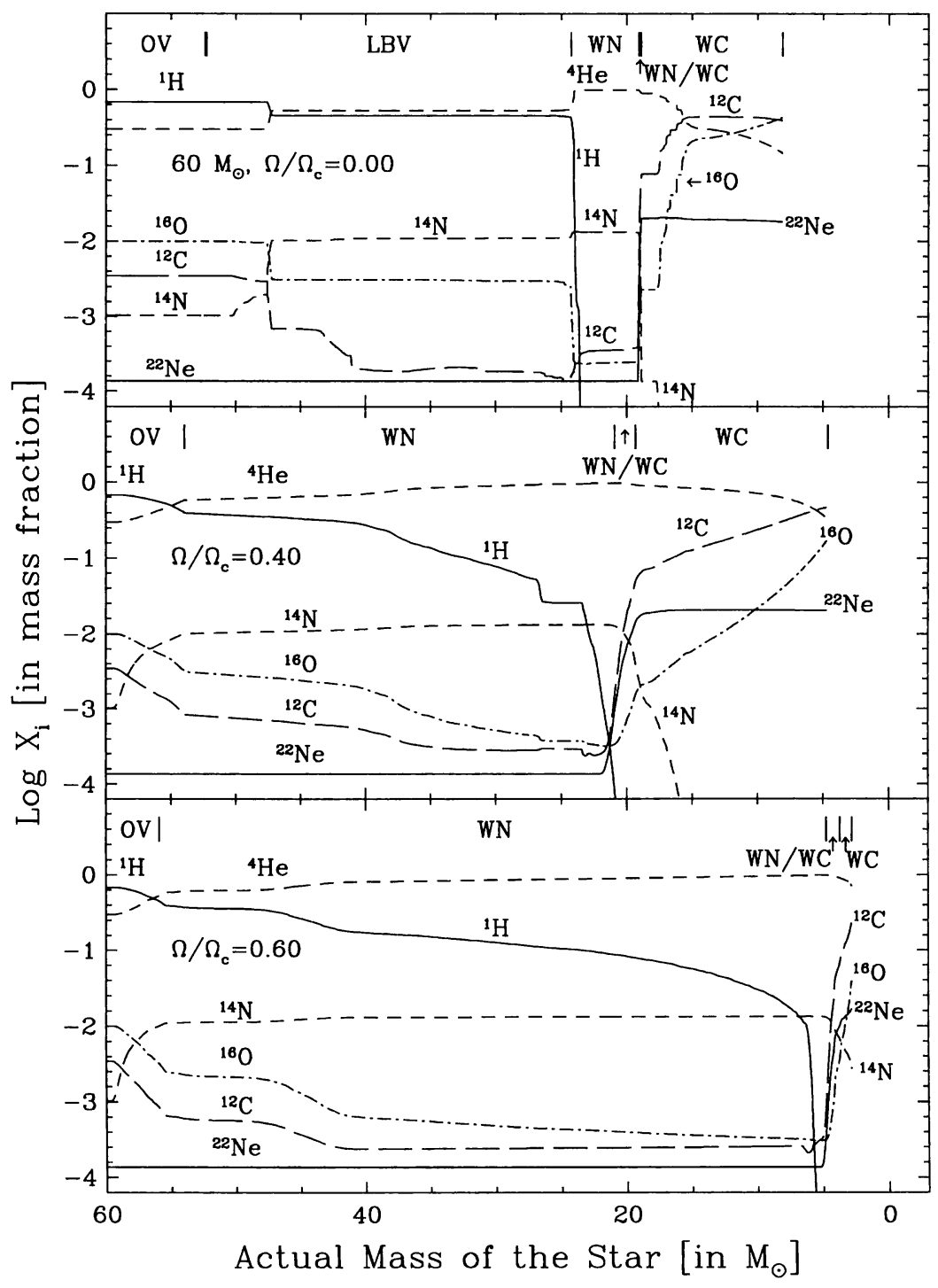

Figure 2. Same as in Fig. 1 for $60 \mathrm{M}_{\odot}$ stellar models at solar metallicity with different initial rotational velocities (see text for more details on the models). $\Omega / \Omega_{c}$ is the angular velocity on the ZAMS divided by the break-up angular velocity. The parts of the evolution during which the star may be considered as an OV main sequence star, as a LBV and as a WR star are indicated. During the WR phase, the WN, the transition WN/WC and the WC phases are distinguished.

occurs for the entrance into the WC phase. Therefore the evolution of the surface abundances are much smoother in rotating models. As a consequence, the $\mathrm{N} / \mathrm{C}$ and $\mathrm{N} / \mathrm{O}$ ratios obtained at the surface of rotating $\mathrm{WN}$ models have not yet 
reached nuclear equilibrium in contrast with the no rotation case where nuclear equilibrium is reached as soon as the star enters the WN phase. One obtains also that rotating models predict longer WN/WC transition phases (Maeder, these Proceedings; see also Langer 1991). One can see in Fig. 2, that during this phase, nitrogen enhancements (H-burning product) can be observed simultaneously with carbon and neon enhancements (He-burning products). The low neon enhancements observed at the surface of the WN8 star WR 147 may well be due to rotational mixing (see Morris et al. in these Proceedings). One notes also (see Fig. 2) that after this transition phase, the ${ }^{22} \mathrm{Ne}$ enhancement reaches more or less the same high level whatever is the initial angular velocity.

One can wonder if the mixture of $\mathrm{H}$ and He-burning products at the surface of transition WN/WC stars is related to some primary nitrogen production. Let us recall that any mixing between the $\mathrm{H}$ - and the He-burning zones would produce primary nitrogen. Indeed primary nitrogen could be produced by the injection of ${ }^{12} \mathrm{C}$ synthesized in the He-burning zone into the $\mathrm{H}$-burning shell. In this last zone, the $\mathrm{CN}$ cycle transforms the ${ }^{12} \mathrm{C}$ into ${ }^{14} \mathrm{~N}$. In fact here this mixing does not occur, because the whole original $\mathrm{H}$-rich envelope has been removed by stellar winds at the time the star enters the He-burning phase.

What are the effects of an increase of the rotation rate on the chemical composition of the WR stellar winds?

(i) As already mentioned above, during the WN phase the processed matter has not yet reached nuclear CNO equilibrium. Moreover, when the rotation rate increases a still greater part of the ejecta becomes rich in H-burning products (long WN phases). Comparisons with Fig. 1 shows that an increase of the mass loss rates tends to increase the quantities of matter ejected during the WC phase ${ }^{2}$.

(2) For the WC phase, Fig. 2 shows that when the initial angular velocity increases, the winds become richer in helium and carbon. In that respect this behaviour is similar to that obtained by an increase of the mass loss rates, however Fig. 2 shows also that the mass ejected during this phase strongly depends on the angular velocity. In particular at high rotation rate, the mass ejected during the WC phase is quite small ${ }^{3}$ and thus the ejecta of the WR star will be mainly dominated by the WN phase.

These remarks above show that an increase of the rotational velocity has different effects compared to an increase of the mass loss rates.

\section{4. ${ }^{26} \mathrm{Al},{ }^{19} \mathrm{~F}$ and ${ }^{22} \mathrm{Ne}$ in Wolf-Rayet stellar winds}

Mass loss by stellar winds enables the star to 'save' some elements from further destruction. In the stellar winds, the abundances are frozen at the state reached at the time of ejection and are no longer modified by nuclear processing. This

\footnotetext{
${ }^{2}$ This is not true at very high metallicity or for very intense stellar winds. In that case the WN phase becomes, as in the case of high rotation rates, more and more important, see Maeder \& Meynet (1994)

${ }^{3}$ The duration may still be important, since when the actual mass of the star decreases, the mass loss rates decrease.
} 
fact may play a role in the process of enrichment of the interstellar medium in ${ }^{26} \mathrm{Al}$ and ${ }^{19} \mathrm{~F}$ by massive stars.

Indeed, in massive stars which become WR stars, ${ }^{26} \mathrm{Al}$ may be synthesized in the H-burning core by ${ }^{25} \mathrm{Mg}$ proton captures and ejected by stellar winds before it is destroyed by neutron captures in the He-burning core (Dearborn $\&$ Blake 1985). Production of ${ }^{26} \mathrm{Al}$ by single non-rotating and non-exploding Wolf-Rayet stars of different masses and metallicities is discussed in Meynet et al. (1996). A discussion of the impact of binarity and of rotation on the ${ }^{26} \mathrm{Al}$ yields is presented by Braun \& Langer (1995).

The observation by the Compton Gamma Ray Observatory of the diffuse $1.8 \mathrm{MeV}$ emission (produced by ${ }^{26} \mathrm{Al}$ decay) implies a present production rate of 1 to $2 \mathrm{M}_{\odot}$ of ${ }^{26} \mathrm{Al}$ per million years in our Galaxy. Since many features of the $1.8 \mathrm{MeV}$ map of the Milky Way favour massive stars (supernovae and/or WR stars) as the main sources for this ${ }^{26} \mathrm{Al}$ (see Roland Diehl, these Proceedings), it is interesting to try to estimate the global contribution of the WR stars in our Galaxy. According to Meynet et al. (1996) (see also Arnould \& Meynet 1997), a total galactic mass of $1.15 \mathrm{M}_{\odot}$ of ${ }^{26} \mathrm{Al}$ might originate solely from non-rotating non-exploding WR stars. Of course, many uncertainties affect this estimate. For different (but still reasonable) values of the IMF, metallicity gradient, SN rate and star formation rate in the Galaxy, we predict that the contribution of the WR stars to the present-day galactic ${ }^{26} \mathrm{Al}$ may range between $0.4-1.3 \mathrm{M}_{\odot}$. The WR stars might thus account for 20 to $70 \%$ of the approximate $2 \mathrm{M}_{\odot}$ of ${ }^{26} \mathrm{Al}$ present nowadays in the Galaxy, and are thus far from being negligible contributors.

${ }^{19} \mathrm{~F}$ is the only stable isotope of fluorine. It is built up at the beginning of the He-burning phase and destroyed at the end of this phase. Again, stellar winds during the WC phase enable the star to remove part of the synthesized ${ }^{19} \mathrm{~F}$ before it is destroyed. According to our stellar models, it appears that this process plays an important role, if not dominant, in ${ }^{19} \mathrm{~F}$ nucleosynthesis (Meynet \& Arnould 1997).

Recently, the strong surface Ne-enrichments predicted by the models of WC stars have been confirmed by the observations (Willis et al. 1996; see also Morris et al. and Dessart et al. in these Proceedings). This fact sets on firmer grounds the role WR stars may play in many different problems. Two of them are briefly discussed below.

The most striking difference between the isotopic composition of the Galactic Cosmic Ray Sources (GCRS) and the solar system is the factor 3-5 enhanced ${ }^{22} \mathrm{Ne} /{ }^{20} \mathrm{Ne}$ ratio observed in the GCRS, while isotopic ratios involving magnesium, silicon and sulfur are the same (Webber et al. 1997). This difference implies that the nucleosynthetic sources which contribute to the isotopic composition of the GCRS are not exactly identical to those which contributed to the isotopic composition of the solar system material. Woosley \& Weaver (1981) suggested that material injected into the Galactic cosmic-rays originate mainly from supernovae with metal-rich progenitors. In that case the model predicts enhancement factors between 1.5 and 2 for all isotopic ratios involving neon, magnesium, silicon and sulfur. If one supposes that for whatever reason ${ }^{4} 5 \%$

\footnotetext{
${ }^{4} \mathrm{~A}$ more detailed model is exposed in Maeder 1984
} 
of the mass injected into the cosmic-rays is WR stellar winds material, then one obtains that the ${ }^{22} \mathrm{Ne} /{ }^{20} \mathrm{Ne}$ ratio is enhanced by a factor 4 while the ratios involving heavier elements remain solar. From this simple consideration, one sees that WR stars seem to be interesting candidates as the source of the most important isotopic anomaly observed in the GCRS.

Let us end this paper by mentioning that the chemical composition of WR stellar winds may have some relevance for understanding some isotopic anomalies observed in meteorites (Arnould et al. 1997a). In particular it appears that some radionuclides have decayed in situ in some meteorites (see the review by Podosek \& Nichols 1997). This fact may be interpreted as resulting from the injection into the proto-solar nebula of these radio isotopes by one or a few nucleosynthetic source(s). It is interesting to mention that such an observation puts some constraints on $\Delta^{*}$, the time elapsed between the last astrophysical event(s) able to affect the composition of the solar nebula and the solidification of some of its material. This time must be shorter than the time required for the decay of the radionuclide if at least part of this latter has decayed in situ in the meteorite.

In the framework of this interpretation, let us wonder to what extent the nucleosynthetic source(s) might have been one or a few WR stars? According to Arnould et al. (1997ab), in the absence of any chemical fractionation between the relevant elements, ${ }^{36} \mathrm{Cl},{ }^{41} \mathrm{Ca}$ and ${ }^{107} \mathrm{Pd}$ can be produced through neutron captures by a variety of WC stars with different initial masses and compositions at a relative level compatible with meteoritic observations ${ }^{5}$. In the case of a $60 \mathrm{M}_{\odot}$ star with solar metallicity, this agreement can be obtained for $\Delta^{*} \simeq 2 \times 10^{5} \mathrm{y}$. It is hoped that in the next years other observational data will permit to better constrain the WR hypothesis. In particular the finding of high ${ }^{205} \mathrm{~Pb}$ excesses would be a distinctive signature of the presence of WR stellar wind material.

\section{Conclusion}

Stellar winds play a key role in the process of enrichment of the interstellar medium in new synthesized elements at least at high metallicity. It may well be that this mode of injection plays also an important role at low metallicity. Firstly the mass loss rates for high mass stars may have a less steep metallicity dependence than the one invoked for smaller initial mass stars. In that respect it is interesting to mention that the differences between the empirical wind momentum versus luminosity relations for galactic, LMC and SMC stars decreases with increasing luminosity (Kudritzki et al. 1995). Moreover if, as suggested by Maeder (these Proceedings), the initial angular velocity of massive stars increases when the metallicity decreases, this will also enhance the importance of mass loss by stellar winds by making WR star formation easier.

Acknowledgments. I express my gratitude to André Maeder for the fruitful collaboration on stellar rotation during the last years.

\footnotetext{
${ }^{5}$ This is also the case for ${ }^{26} \mathrm{Al}$, but this element is not produced by neutron capture.
} 


\section{References}

Arnould, M., Meynet, G. 1997, in: C. Winkler et al. , The Transparent Universe, Proc. 2nd INTEGRAL Workshop, ESA SP-382, p. 33

Arnould, M., Meynet, G., Paulus, G. 1997a, in: T.J. Bernatowicz \& E. Zinner (eds.), Astrophysical Implications of the Laboratory Study of Presolar Material, AIPCP 402, p. 179

Arnould, M., Paulus, G., Meynet, G. 1997b, A\&A 321, 452

Braun, H., Langer, N. 1995, in: K.A. van der Hucht \& P.M. Williams (eds.), WolfRayet Stars: Binaries, Colliding Winds, Evolution, Proc. IAU Symp. No. 163, (Dordrecht: Kluwer), p. 305

Charbonnel, C., Meynet, G., Maeder, A., Schaller, G., Schaerer, D. 1993, A\&AS 101, 415

Dearborn, D.S.P., Blake, J.B. 1985, ApJ 288, L21

Fliegner, J., Langer, N. 1994, in: K.A. van der Hucht \& P.M. Williams (eds.), WolfRayet Stars: Binaries, Colliding Winds, Evolution, Proc. IAU Symp. No. 163, (Dordrecht: Kluwer), p. 326

Friend, D.B., Abbott, D.C 1986, ApJ 311, 701

Kudritzki, R.P., Lennon, D.J., Puls, J. 1995, in: J.R. Walsh \& I.J. Danziger (eds.), Science with the VLT, p. 246

Lamers, H., Cassinelli, J.P. 1996, in: C. Leitherer, U. Fritze-von Alvensleben \& J. Huchra (eds.), From Stars To Galaxies: The Impact of Stellar Physics on Galaxy Evolution, ASP-CS 98, 162

Langer, N. 1991, A\&A 248, 531

Maeder, A. 1984, Adv. Space Res. 4, No. 2-3, 55

Maeder, A. 1991, A\&A 242, 93

Maeder, A. 1992, A\&A 264, 105

Maeder, A. 1993, A\&A 268, 833

Maeder, A., Meynet G. 1994, A\&A 287, 803

Meynet, G., Arnould, M. 1997, in: J.-M. Vreux, A. Detal, D. Fraipont-Caro, E. Gosset $\&$ G. Rauw (eds.), Wolf-Rayet Stars in the Framework of Stellar Evolution, Proc. $33^{\text {rd }}$ Liège Int. Astroph. Coll. (Liège: Univ. of Liège), p. 89

Meynet, G., Arnould, M., Prantzos, N., Paulus, G. 1997, A\&A 320, 460

Podosek, F.A., Nichols, R.H. 1997, in: T.J. Bernatowicz \& E. Zinner (eds.), Astrophysical Implications of the Laboratory Study of Presolar Material, AIP-CP 402, p. 617

Prantzos, N., Vangioni-Flam, E., Chauveau, S. 1994, A\&A 285, 132

Rayet, M.R., Hashimoto, M. 1998, in: M. Arnould et al. (eds.), Tours Symposium on Nuclear Physics III, AIP-CP 425, 605

Schaerer, D., Meynet, G., Maeder, A., Schaller, G. 1993, A\&AS 98, 523

Schaller, G., Schaerer, D., Meynet, G., Maeder, A. 1992, A\&AS 96, 269

Smith, L.F., Maeder, A. 1991, A\&A 241, 77

Webber, W.R., Lukasiak, A., McDonald, F.B. 1997, ApJ 476, 766

\section{Discussion}

Vanbeveren: (1) Can you make a guess how the yields will change, if you would apply stellar wind mass loss rates of WR stars a factor two smaller as suggested yesterday? 
(2) André Maeder (1992) suggested that at the end of their life most of the massive stars with $M_{i} \lesssim 20-25 \mathrm{M}_{\odot}$ collapse completely and form black holes (no SN explosion). Are you still following this idea?

Meynet: (1) At a given metallicity, when the mass loss rate decreases, the global ejecta (those of the WR and of the SN) would be poorer in helium and carbon and richer in oxygen. (2) Models with low rotation rate behave like non-rotating models, thus the result you mention remains still valid for low rotation rate. The evolution of a stellar population has to be convolved with the angular velocity distribution, and a lot of stellar models for different initial masses and angular velocities have to be computed before we will be able to appreciate the effects of rotation on the problem you mention.

Langer: I did not understand whether your results on the ${ }^{19} \mathrm{~F}$ production included the effect of rotation or not. I am asking because we find that rotational mixing does greatly enhance the ${ }^{19} \mathrm{~F}$ yield in $10-30 \mathrm{M}_{\odot}$ stars. Can you confirm this effect?

Meynet: The results concerning the ${ }^{19} \mathrm{~F}$ production by WR stars were obtained with non-rotating stellar models, but I expect that mixing would enhance ${ }^{19} \mathrm{~F}$ production by bringing protons in the He-burning region.

van der Hucht: Do I understand correctly that rotation delays the appearance of the Ne-overabundance in the evolution of WN $\rightarrow$ WC stars? ISO-sWs high-resolution spectroscopy of a WN9o (no-hydrogen) star shows a [NeIII $15.5 \mu \mathrm{m}$ emission-line flux indicative of an Ne-overabundance of a factor $\sim 2$. Would this be inconsistent with your evolution-with-rotation models?

Meynet: Rotation considerably enlarges the range of possible values of the Ne-overabundance during the transition WN/WC phase. This is in contrast with non-rotating models, where the overabundance of $\mathrm{Ne}$ appears in a very short interval of time and where there is no possibility to observe an intermediate overabundance of neon. After this transition phase, the Ne-overabundance in rotating models is of the same order as in non-rotating stellar models. Therefore, as far as the star you mention can be considered to be in this transition phase, rotation might be an explanation for its Neoverabundance.

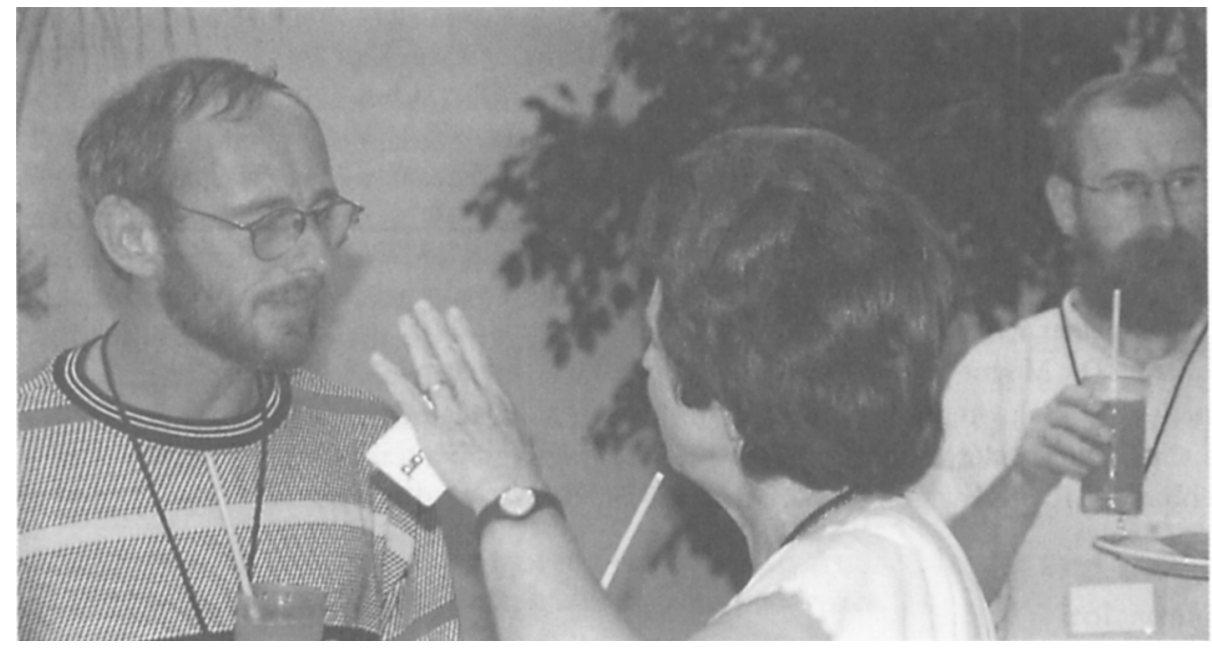

